University of Wollongong

Research Online

Faculty of Social Sciences - Papers (Archive) Faculty of Arts, Social Sciences \& Humanities

$1-1-2012$

Refractive error and monocular viewing strengthen the hollow-face illusion

Harold C. Hill

University of Wollongong, harry@uow.edu.au

Stephen A. Palmisano

University of Wollongong, stephenp@uow.edu.au

Harold Matthews

University of Wollongong

Follow this and additional works at: https://ro.uow.edu.au/sspapers

Part of the Education Commons, and the Social and Behavioral Sciences Commons

Research Online is the open access institutional repository for the University of Wollongong. For further information contact the UOW Library: research-pubs@uow.edu.au 


\title{
Refractive error and monocular viewing strengthen the hollow-face illusion
}

\begin{abstract}
We measured the strength of the hollow-face illusion-the 'flipping distance' at which perception changes between convex and concave-as a function of a lens-induced 3 dioptre refractive error and monocular/ binocular viewing. Refractive error and closing one eye both strengthened the illusion to approximately the same extent. The illusion was weakest viewed binocularly without refractive error and strongest viewed monocularly with it. This suggests binocular cues disambiguate the illusion at greater distances than monocular cues, but that both are disrupted by refractive error. We argue that refractive error leaves the ambiguous low-spatial-frequency shading information critical to the illusion largely unaffected while disrupting other, potentially disambiguating, depth/distance cues. 2012 a Pion publication.
\end{abstract}

\section{Keywords}

strengthen, hollow, refractive, face, error, illusion, monocular, viewing

Disciplines

Education | Social and Behavioral Sciences

\section{Publication Details}

Hill, H. C., Palmisano, S. A. \& Matthews, H. (2012). Refractive error and monocular viewing strengthen the hollow-face illusion. Perception, 41 (10), 1281-1285. 
Refractive error and monocular viewing strengthen the hollow-face illusion

Harold Hill*, Stephen Palmisano and Harold Matthews

School of Psychology, University of Wollongong, Wollongong, NSW 2522, Australia

*Corresponding author

harry@uow.edu.au

Tel. +61 42214073

Fax. +6142214163

\section{Abstract}

We measured the strength of the hollow-face illusion - the 'flipping distance' at which perception changes between convex and concave - as a function of a lens induced three-dioptre refractive error and monocular/binocular viewing. Refractive error and closing one eye both strengthened the illusion to approximately the same extent. The illusion was weakest viewed binocularly without refractive error and strongest viewed monocularly with. This suggests binocular cues disambiguate the illusion at greater distances than monocular cues but that both are disrupted by refractive error. We argue that refractive error leaves the ambiguous low spatial frequency shading information critical to the illusion largely unaffected while disrupting other, potentially disambiguating, depth/distance cues. [110 words]

Keywords: Hollow-face illusion, refractive error, depth perception, depth reversal, shading 
The hollow-face illusion is the perception of a concave mask as a convex face when viewed from beyond a certain distance. The distance at which perception changes between convex and concave, the 'flipping distance', can be used as a measure of the strength of the illusion and has been shown to vary as a function of a variety of viewing conditions including lighting direction, monocular/binocular viewing and the orientation of the mask (Hill and Bruce 1993). Here we investigated whether the refractive error associated with wearing positive three-dioptre reading glasses also affects flipping distance. Wearing three-dioptre lenses brings the resting focal length of the eye to $1 / 3$ metres and, assuming the lens cannot be flattened further, means that the image of any object beyond that distance will be blurred. This blurring of the retinal image will disrupt a number of depth cues that may be important in disambiguate the mask at closer distances. For example, adding blur reduces both accommodative accuracy (Heath 1956) and the ability to discriminate differences in blur (Mather and Smith 2002), both potential cues to surface layout at least when integrated across eye movements. Blur is also in some ways equivalent to a greater viewing distance in that both limit the high spatial frequency information available.

The inherently ambiguous pattern of shading - a concave surface lit from one direction will produce roughly the same pattern of shading as a convex surface lit from the opposite direction (at least with regards its Lambertian component) - is thought to be central to the illusion. An opaque mask lit from in front and below looks like a convex face lit from above. The convex percept is consistent with a "light-from-above" assumption (Adams et al 2004; O'Shea et al, 2010; Sun and Perona, 1998). The hollow-face illusion is stronger when the convex percept appears lit from above (Hill and Bruce 1993), as is also the case for a translucent mask lit from above and behind as used here. As shading contains predominantly low spatial frequency (Morrison and Schyns 2001), we predicted that refractive-error-induced blurring might strengthen the illusion by preserving ambiguous low spatial frequency cues at the same time as degrading unambiguous cues to the mask's true depth. Consistent with this, it has previously been shown that we converge on the illusory face rather than the actual mask and suggested that this may "make it easier to maintain the illusion by defocusing the image" (Hoffmann and Sebald 2007, p.469).

We do not know what visual information disambiguates the mask at closer distances but binocular disparities, vergence, accommodation, image blur (if combined over eye movements), and motion parallax (if a stationary object is assumed ${ }^{1}$ ) may all be involved individually or in combination. Monocular viewing is known to strengthen the illusion (Hill and Bruce 1993), suggesting that unambiguous binocular information based on binocular disparities and/or convergence disambiguate the illusion at greater distances than monocular cues. If refractive error selectively disrupts binocular information, for example by limiting stereo resolution (Banks et al 2004), we would expect refractive error to have a greater effect on binocular than monocular viewing. However, even monocularly the mask appears hollow when viewed from close enough showing that monocular cues can disambiguate the illusion albeit at shorter distances. While pictorial cues are inherently ambiguous due to the limitations of a single line of sight, accommodation and parallax from self motion are potentially disambiguating monocular cues. Built-

\footnotetext{
${ }^{1}$ The compelling impression that the hollow-face and related illusions of depth reversal 'follow' you when you move from side-to-side suggest that the visual system does not incorporate such an assumption (Rogers and Gyani 2010)
} 
in 'assumptions', for example that of equally sized elements for relative size or shape-from-texture, mean that even for pictorial cues certain percepts are more likely and this could help disambiguate the illusion when those assumptions are met. If refractive error disrupts whatever monocular cues disambiguate the illusion, it will increase the strength of the illusion viewed monocularly as well as binocularly.

In order to test these possibilities we measured flipping distance when approaching the illusion when viewing with one (the dominant) or both eyes and when either wearing or not wearing three-dioptre reading glasses. The mask was positioned $1.25 \mathrm{~m}$ above the ground and lit from above and behind using an angle-poise light with a cool daylight bulb (please see Figure 1). Twenty-one observers took part in the 2 Refractive Error (Zero/Three Dioptres) $\times 2$ Viewing (Monocular/binocular) within -subjects design. All observers had 10/20 or better acuity measured using a Snellen eye chart viewed from a distance of 10 feet. Order of trials was randomised for each observer with one repetition for each condition.

a)
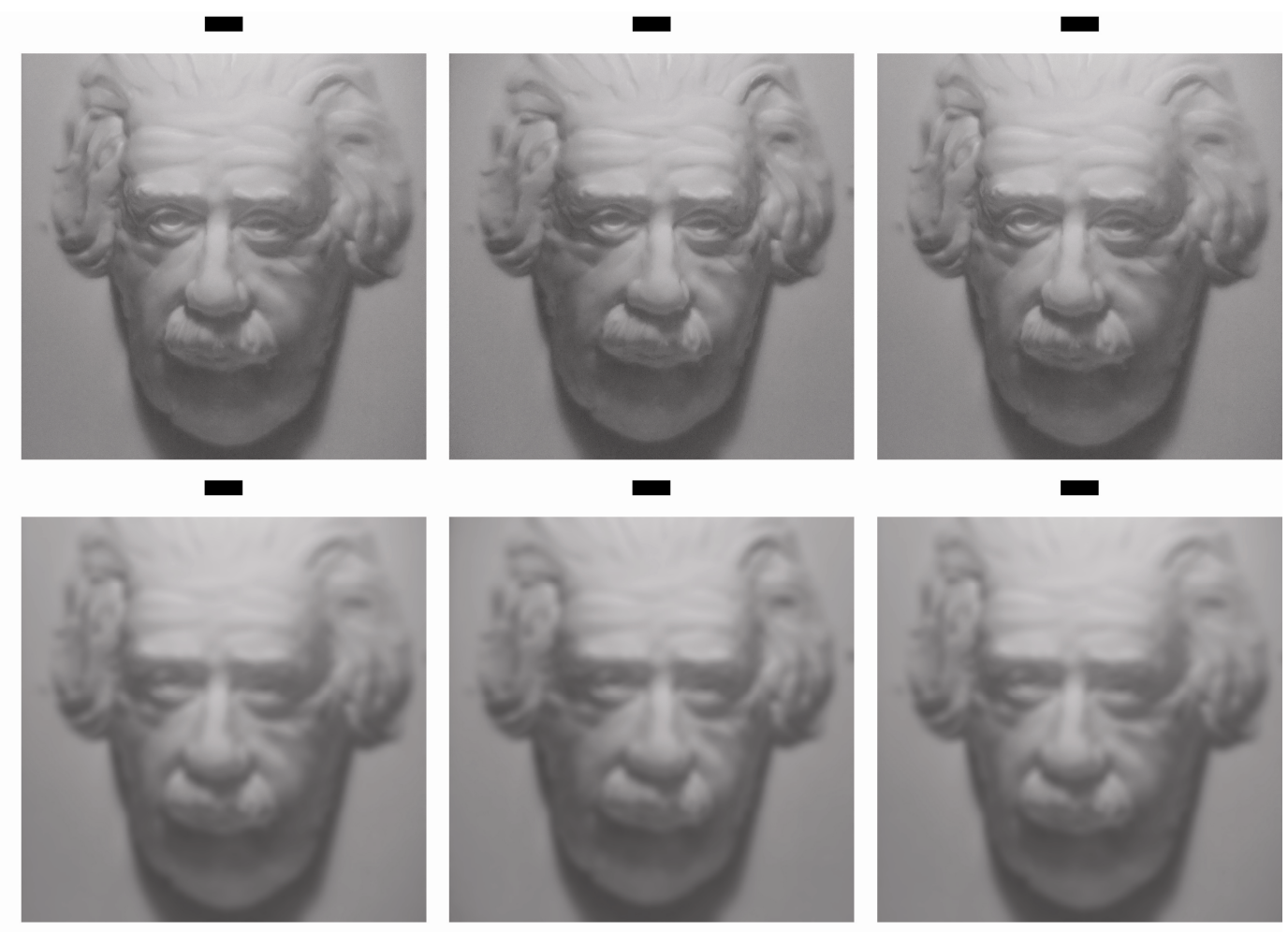

$\mathrm{L}$

$\mathrm{R}$

$\mathrm{L}$

Figure 1 illustrates the stimulus used in the experiment. All images show the mask taken with a stereo camera (Fujifilm W1) from a distance of $50 \mathrm{~cm}$. The top row shows unfiltered images whereas the bottom row has been blurred with "lens blur" in Adobe Photoshop to approximate the appearance of the mask when viewed with refractive error. It may be difficult or impossible to see even stereoscopic photographs of the hollow-face as hollow, but left pairs correspond to the hollow face for parallel fusion and the right pairs for cross fusion.

As illustrated in Figure 2, the illusion was weakest (flipping distance largest) when viewed binocularly without refractive error and strongest (smallest flipping distance) when viewed monocularly with refractive error. Inducing a three-dioptre refractive error or closing one eye 
strengthened the illusion to approximately the same extent (both approximately halved the flipping distance). Data was right skewed and leptokurtic but robustness was initially assumed. ANOVA gave a significant two-way interaction between refractive error and monocular/binocular viewing, $F(1,20)$ $=15.26, p=.001, \eta_{p}{ }^{2}=.43$. Simple main effects analysis showed significant effects of refractive error for both binocular, $F(1,40)=46.98, p<.001, \eta_{p}{ }^{2}=.54$, and monocular viewing, $F(1,40)=6.63, p=.014$, $\eta_{p}{ }^{2}=.14$. In order to confirm that this result was not an artefact of the non normal data, we used Monte Carlo resampling of the participant data permuting the scores in each condition to test the same null hypotheses (Berkovits et al 2000). Based on 5000 iterations, the $p$ value for the refractive error $x$ viewing condition was $p<.001$. There were also significant effects of refractive error for both monocular and binocular viewing. These results are both entirely consistent with the parametric analysis.

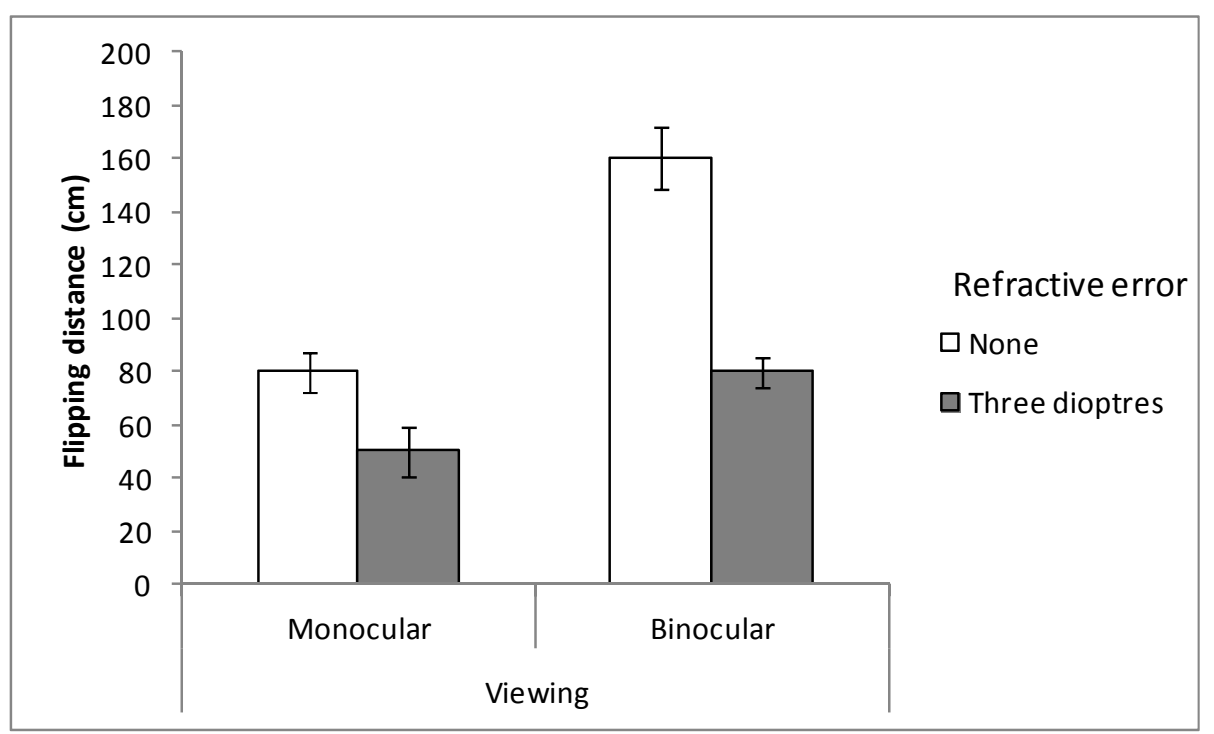

Figure 2. Mean 'flipping distance' for monocular or binocular viewing, with or without a threedipotre refractive error. Error bars show standard error of the mean after removal of between subjects variation.

Artificially introducing a three-dioptre refractive error and monocular viewing both strengthened the illusion. Figure 2 and partial eta-squared values suggest that refractive error and closing one eye produce effects of similar sizes. There was an effect of refractive error on the illusion viewed monocularly. The interaction suggests that the effects are not additive and that both affect overlapping information, but may result from a floor effect.

We do not know which of the available depth cues are responsible for resolving the illusion at close distances as the magnitudes of many if not all possible cues will increase as viewing distance decreases. The issue is further complicated as the relative contribution of different cues may also vary with distance. However the effect of monocular as compared to binocular viewing reported here and previously suggests that binocular cues disambiguate the illusion at greater distances than monocular ones. Both horizontal and vertical (Gillam et al 1988) disparities are potentially involved, but because the visual angle subtended by the mask was typically less than $15^{\circ}$ and the mask was presented medially, any vertical disparity gradients will be minimal and thus ineffective as a source of information about absolute distance (Bradshaw, Glennerster \& Rogers, 1996). Vergence may be important and there is evidence for its use as a cue to distance within one metre (Tresilian et al 
1999). If people tend to verge on the illusory rather than the actual nose (Hoffmann and Sebald 2007) a change in vergence would be expected to accompany 'flipping' but remains to be demonstrated. Whatever binocular cues do disambiguate the illusion at $\sim 160 \mathrm{~cm}$, refractive error makes them less effective. Refractive error would reduce the precision of both horizontal and vertical disparities but would not affect the angles of the converging lines of sight from each eye. Vergence may be affected due to its dependence on detecting the correct alignment of the image as well as its links with accommodation and blur.

Monocular cues do disambiguate the illusion at close distances. Motion parallax from observer bob and sway is one potentially unambiguous monocular cue given a stationary object assumption but, as noted earlier, does not appear to be involved in practice. While participants were encouraged to move directly towards the mask and to only sway after experiencing a flip as a check that the now concave mask no longer 'followed' them, this was not tightly controlled. In principle, accommodation provides absolute distance information and would vary within the range of flipping distances reported. It may provide ordinal depth information between $17 \mathrm{~cm}$ and $50 \mathrm{~cm}$ (Fisher and Ciuffreda 1988; Mon-Williams and Tresilian 2000). The backlit plastic mask used is likely to be a poor accommodative stimulus given its lack of sharp contours and this may contribute to its effectiveness as an illusion. Positive three-dioptre lenses would not be expected to elicit an accommodative response given the stimulus is beyond rather than in front of the focal point (Fincham 1951) making the loss of accommodation a possible basis of the effect of refractive error in the monocular condition. However, even in the monocular condition with refractive error the average flipping distance of $50 \mathrm{~cm}$ was greater than the $33 \mathrm{~cm}$ focal length of the lenses used making it unlikely that 'the flip' was associated with an accommodative response. Whatever monocular cues disambiguate the mask at close distances, the results show their effective distance is reduced by refractive error. Two participants reported still seeing the mask as convex even at the closest possible distance (their nose inside the mask) for monocular viewing with or without refractive error (flipping distance was recorded as zero centimetres).

We initially thought that natural refractive error might explain some of the individual variation in susceptibility to the hollow-face illusion found here and elsewhere - here differences between observers accounted for $45.8 \%$ of total variance. However we did not find any correlation between measured acuity for different participants and their flipping distance in the zero refractive error (or any other) condition as would be expected if this was the case (all p's >.3). It has also been reported that people with schizophrenia are not susceptible to the illusion (Dima et al 2009) and low spatial frequency sensitivity decrements also associated with this disorder (O'Donnell et al 2002) might help to explain this as well as other aspects of their face processing (Silverstein et al 2009). Low spatial frequencies may also be particularly important for initiating top-down processes in object recognition (Bar et al 2006), processes that are likely to contribute to the strength of the hollow-face illusion in "normals" but that may again be impaired for people with schizophrenia.

Three-dioptre refractive error, like monocular viewing, increased the distance over which observers reported seeing the hollow-face illusion probably because refractive error preserves ambiguous low spatial frequency shading while degrading other monocular and binocular cues to actual depth [ words].

Acknowledgments. 
Thank you to the students who took part in the experiment and to two anonymous reviewers who made many helpful and constructive suggestions as well as pointing out our earlier errors. The work was supported in part by Discovery Project 0986898 from the Australian Research Council. 


\section{References}

Adams W J, Graf E W, Ernst M O, 2004 "Experience can change the 'light-from-above' prior" Nature Neuroscience 7 1057-1058

Banks, M S, Gepshtein, S, Landy, M S, 2004 Why is spatial stereoresolution so low? (Washington, DC, ETATS-UNIS: Society for Neuroscience)

Bar M, Kassam K S, Ghuman A S, Boshyan J, Schmid A M, Dale A M, Hämäläinen M S, Marinkovic K, Schacter D L, Rosen B R, Halgren E, 2006 "Top-down facilitation of visual recognition" Proceedings of the National Academy of Sciences of the United States of America 103 449454

Berkovits I, Hancock G R, Nevitt J, 2000 "Bootstrap Resampling Approaches for Repeated Measure Designs: Relative Robustness to Sphericity and Normality Violations" Educational and Psychological Measurement 60 877-892

Bradshaw, M F, Glennerster, A, Rogers, B J (1996) "The effect of size on disparity scaling from differential perspective and vergence cues" Vision Research 36, 1255-1264

Dima D, Roiser J P, Dietrich D E, Bonnemann C, Lanfermann H, Emrich H M, Dillo W, 2009 "Understanding why patients with schizophrenia do not perceive the hollow-mask illusion using dynamic causal modelling" Neurolmage 46 1180-6

Fincham E F, 1951 "The accommodation reflex and its stimulus" The British journal of ophthalmology $35381-93$

Fisher S K, Ciuffreda K J, 1988 "Accommodation and apparent distance" Perception 17 609-621

Gillam B, Chambers D, Lawergren B, 1988 "The role of vertical disparity in the scaling of stereoscopic depth perception: An empirical and theoretical study" Attention, Perception, \& Psychophysics 44 473-483

Heath G G, 1956 "The influence of visual acuity on accommodative responses of the eye" American Journal of Optometry \& Archives of American Academy of Optometry 33 513-524

Hill H, Bruce V, 1993 "Independent effects of lighting, orientation, and stereopsis on the hollow-face illusion" Perception 22 887-97

Hoffmann J, Sebald A, 2007 "Eye vergence is susceptible to the hollow-face illusion" Perception 36 461-470

Mather G, Smith D R R, 2002 "Blur discrimination and its relation to blur-mediated depth perception" Perception 31 1211-1219

Mon-Williams M, Tresilian J R, 2000 "Ordinal depth information from accommodation?" Ergonomics 43 391-404

Morrison D, Schyns P, 2001 "Usage of spatial scales for the categorization of faces, objects, and scenes" Psychonomic Bulletin \& Review 8 454-469

O'Donnell B F, Potts G F, Nestor P G, Stylianopoulos K C, Shenton M E, McCarley R W, 2002 "Spatial Frequency Discrimination in Schizophrenia" Journal of Abnormal Psychology 111 620-625

O'Shea J P, Agrawala M, Banks M S, 2010 "The influence of shape cues on the perception of lighting direction" Journal of Vision 10

Tresilian J R, Mon-Williams M, Kelly B M, 1999 "Increasing confidence in vergence as a cue to distance" Proceedings of the Royal Society of London. Series B: Biological Sciences 266 39-44

Rogers B, Gyani A, 2010 "Binocular disparities, motion parallax, and geometric perspective in Patrick Hughes's 'reverspectives': Theoretical analysis and empirical findings" Perception 39 330-348

Silverstein S M, All S D, Kasi R, Berten S, Essex B, Lathrop K L, Little D M, 2009 "Increased fusiform area activation in schizophrenia during processing of spatial frequency-degraded faces, as revealed by fMRI" Psychological Medicine 40 1159-1169

Sun J, Perona P, 1998 "Where is the sun?" Nature Neuroscience 1 183-184 